\title{
Bounds and estimates on eddy current losses in soft magnetic composites
}

Cite as: J. Appl. Phys. 123, 235109 (2018); https://doi.org/10.1063/1.5031128

Submitted: 28 March 2018 . Accepted: 05 June 2018 . Published Online: 21 June 2018

Xiaotao Ren (D), Romain Corcolle, and Laurent Daniel

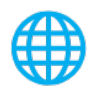

\section{ARTICLES YOU MAY BE INTERESTED IN}

Past, present, and future of soft magnetic composites

Applied Physics Reviews 5, 031301 (2018); https://doi.org/10.1063/1.5027045

New Soft Magnetic Composites for electromagnetic applications with improved mechanical properties

AIP Advances 6, 056209 (2016); https://doi.org/10.1063/1.4943413

Improving the mechanical quality factor of ultra-low-loss silicon resonators

Journal of Applied Physics 123, 235105 (2018); https://doi.org/10.1063/1.5027486

\section{Lock-in Amplifiers up to $600 \mathrm{MHz}$}
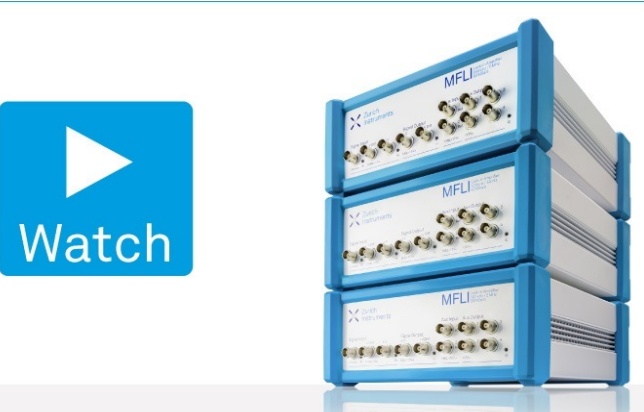


\title{
Bounds and estimates on eddy current losses in soft magnetic composites
}

\author{
Xiaotao Ren, ${ }^{1}$ Romain Corcolle, ${ }^{1,2, a)}$ and Laurent Daniel ${ }^{1}$ \\ ${ }^{1}$ GeePs | Group of Electrical Engineering-Paris, UMR CNRS 8507, CentraleSupélec, Univ. Paris-Sud, \\ Université Paris-Saclay, Sorbonne Universités, UPMC Univ Paris 06, 3 \& 11 rue Joliot-Curie, \\ Plateau de Moulon, 91192 Gif-sur-Yvette Cedex, France \\ ${ }^{2}$ New York University Shanghai, Shanghai 200122, China
}

(Received 28 March 2018; accepted 5 June 2018; published online 21 June 2018)

\begin{abstract}
Soft Magnetic Composites can be considered as a periodic pattern of circular fibers or spheres inside a matrix. In this paper, lower and upper bounds for eddy current (EC) losses are analytically deduced for these types of microstructures. Bounds are obtained from simple magnetic field averaging operations within the inclusions. The averaging manipulations rely on the determination of the effective permeability of the composite, which can be estimated using a homogenization strategy. Lower and upper estimates of EC losses are then obtained for more generic microstructures based on the definition of this effective permeability. Results are compared to Finite Element calculations. The model is then validated with experimental results from the literature. Published by AIP Publishing. https://doi.org/10.1063/1.5031128
\end{abstract}

\section{INTRODUCTION}

Soft Magnetic Composites (SMCs) are magnetic materials typically composed of ferromagnetic inclusions embedded in a dielectric matrix. They are designed to reduce Eddy Current (EC) losses while maintaining high magnetic permeability. This can be achieved with highly magnetic grains insulated by dielectrics, which cut down the global eddy currents. The grains are chosen as small as possible so as to obtain minimum EC losses. ${ }^{1}$ In recent research works on SMCs, the typical grain size is of the order of $100 \mu \mathrm{m},{ }^{2-5}$ but many efforts are devoted to reduce it. $^{6-8}$ Modeling completely such materials, including their microstructure, in a numerical design tool is usually impossible due to the large scale difference between the gain size and the typical device size. For such multi-scale problems, the use of homogenization strategies is a preferable choice to describe the macroscopic behavior. The main properties of interest in SMCs are the effective magnetic permeability and the EC loss density.

A classical approximation for the modeling of SMCs is to consider the microstructure as a periodic matrix/inclusion microstructure. ${ }^{9-11}$ Several approaches can be found to estimate the effective electromagnetic properties of composite materials. ${ }^{12-14}$ As for EC losses, numerical ${ }^{10,15}$ and analyti$\mathrm{cal}^{16}$ approaches have been proposed for 2D SMCs (considering infinitely long cylinder inclusions for the third dimension) with applied field in the perpendicular direction (cylinder axis). Under such conditions at low frequency, the magnetic field in the whole domain is uniform, which greatly simplifies the problem since EC losses can be related to the square of the uniform magnetic field in the conductive inclusion.

Nevertheless, considering in-plane field for such microstructures is much more challenging. The reason is that the field distribution in the inclusions cannot be considered

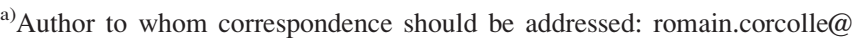
nyu.edu
}

uniform anymore. In the case of spherical inclusions arranged periodically, the same problem arises. An option is to replace the magnetic field in the magnetic inclusions by its mean value, ${ }^{17}$ which can be determined analytically or numerically. This approach often provides an estimate of EC losses with a satisfying accuracy.

The results obtained from that approach showed that EC losses were systematically underestimated. This observation brought the suspicion that a lower bound for EC losses was obtained, but it was not proved. This paper demonstrates that using this strategy for a square lattice of cylindrical inclusions or a periodic cubic lattice of spherical inclusions indeed provides a lower bound for EC losses. Moreover, another approach that replaces the distorted magnetic field by its second order moment (average of the square of the magnetic field) ${ }^{18}$ is proved to provide an upper bound on the EC losses. The approach is then extended to a more general class of microstructures for SMCs. It is shown that, although the bound status cannot be guaranteed, the method still provides lower and upper estimates for the EC losses.

In the first part of the paper, EC loss density is defined and a generic formula for SMCs with cylindrical or spherical inclusions is given. The second part is dedicated to the analytical derivation of lower and upper bounds on EC loss density for cylinder or sphere microstructures. The method is then extended to more generic microstructures. EC loss density estimates are given and compared to Finite Element Method (FEM) results. The proposed model is discussed for SMCs with randomly distributed inclusions, and it is finally applied to a practical configuration and compared to experimental results from the literature.

\section{EDDY CURRENT LOSSES}

\section{A. Definition of EC loss density}

According to Faraday's law, a time varying magnetic field generates an electric field $\mathbf{E}$. In a domain $\Omega$ with 
electric conductivity $\sigma$, eddy current $\mathbf{J}_{E C}=\sigma \mathbf{E}$ arises, which further results in Joule losses in the material. In the harmonic regime, the EC loss density $\mathcal{U}$ is defined as the Joule losses dissipated per unit volume during a wave period

$$
\mathcal{U}=\frac{\left\langle\mathbf{E}^{t} \sigma \mathbf{E}\right\rangle}{2 f}
$$

where $f$ is the frequency and the operator $\langle\cdot\rangle$ denotes a volume average over the domain $\Omega$ by $\langle\cdot\rangle=\frac{1}{\Omega} \int_{\Omega} \cdot \mathrm{d} \Omega$ and $[\cdots]^{t}$ indicates a conjugate transpose operator.

Assuming linear electromagnetic behavior, the induced electric field $\mathbf{E}$ in the domain can be deduced using the following Maxwell equations in the harmonic regime:

$$
\left\{\begin{array}{l}
\nabla \times \mathbf{E}=-j \omega \mu \mathbf{H} \\
\nabla \times \mathbf{H}=\sigma \mathbf{E}+j \omega \epsilon \mathbf{E},
\end{array}\right.
$$

where $\mu$ denotes the magnetic permeability, $\epsilon$ the electric permittivity, and $\omega$ the angular frequency $(\omega=2 \pi f)$. These equations enable to determine the electric and magnetic field distributions.

Magnetic field $\mathbf{H}$ can be mathematically split into two contributions $\mathbf{H}=\mathbf{H}_{q s}+\mathbf{H}_{E C}, \mathbf{H}_{q s}$ representing the magnetic field in quasistatics and $\mathbf{H}_{E C}$ representing the induced magnetic field due to EC. At low frequency, $\mathbf{H}_{E C}$ can be neglected, simplifying the first line of (2) into

$$
\nabla \times \mathbf{E}=-j \omega \mu \mathbf{H}_{q s} .
$$

\section{B. Eddy current losses in elementary cells of SMCs}

SMCs consist of inclusions surrounded by an insulating matrix. The effective permeability $\tilde{\mu}$ of this heterogeneous material can be estimated using a homogenization strategy. $\tilde{\mu}$ then links the macroscopic magnetic field $\overline{\mathbf{H}}$ and the macroscopic flux density $\overline{\mathbf{B}}$

$$
\overline{\mathbf{B}}=\tilde{\mu} \overline{\mathbf{H}} .
$$

In this work, attention is first focused on two simplified microstructures: square lattice of fiber inclusions and cubic lattice of spherical inclusions, as shown in Fig. 1. The fiber inclusion problem can be reduced to a 2D study.

First, consider the study of a single cell of SMCs with a cylindrical or a spherical inclusion with conductivity $\sigma_{i}$ and permeability $\mu_{i}$ (linear isotropic constituent). If the magnetic field $\mathbf{H}_{i}$ in the inclusion is uniform, the EC loss density has the following simple expression: ${ }^{17}$

$$
\mathcal{U}=\xi \pi f \sigma_{i} \mu_{i}^{2} \mathbf{K}^{t} \cdot\left[\begin{array}{c}
H_{i x}^{2} \\
H_{i y}^{2} \\
H_{i z}^{2}
\end{array}\right],
$$

where $\xi$ is the volume fraction of the inclusion (filling factor). Shape factor vector $\mathbf{K}$ value depends on the shape and size of the inclusion ${ }^{17}$

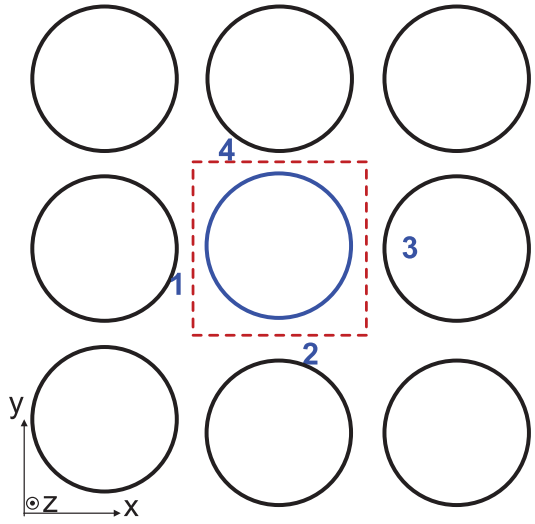

(a)

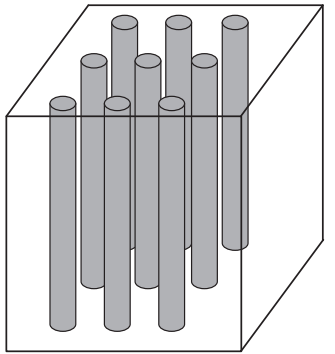

(b)

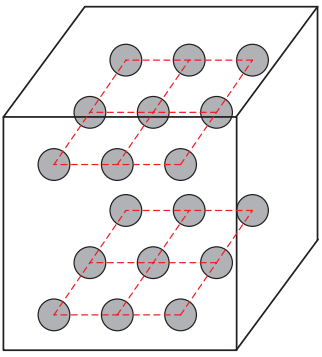

(c)
FIG. 1. (a) 2D sketch of cubic lattice of spherical inclusions or square lattice of fiber inclusions. The domain confined by dashed lines 1-4 forms an elementary cell of periodic pattern. (b) 3D view of square lattice of fiber inclusions (case 1). (c) 3D view of cubic lattice of spherical inclusions (case 2).

$$
\mathbf{K}^{t}= \begin{cases}{\left[\frac{1}{2}, \frac{1}{2}, \frac{1}{4}\right] \pi R^{2}} & \text { (cylindrical inclusion) } \\ {\left[\frac{1}{5}, \frac{1}{5}, \frac{1}{5}\right] \pi R^{2}} & \text { (spherical inclusion) }\end{cases}
$$

with $R$ the radius of a cylinder or a sphere.

For SMCs, the magnetic field in the inclusions is usually nonuniform and (5) is not valid anymore. Then, (1) cannot be expressed as a function of the magnetic field distribution in such a simple manner. Moreover, a complete determination of the magnetic field distribution in the inclusions can be rather complex, if not impossible. A simpler approach is to use statistical information about the field distribution. Mean-field approaches providing effective permeability enable to retrieve directly such pieces of information: average and second-order moment of magnetic field in the inclusions. ${ }^{19}$

Then, based on (5), two estimates of EC loss density can be built. The components $H_{i m}^{2}(m=x, y, z)$ in (5) can be replaced by the first or second order moment of the magnetic field, $\left\langle H_{m}\right\rangle_{i}^{2}$ and $\left\langle H_{m}^{2}\right\rangle_{i}$ respectively. $\langle\cdot\rangle_{i}$ denotes an average operator over the inclusion $i$. This leads to the following two estimates for EC loss density, noted $\mathcal{U}^{-}$and $\mathcal{U}^{+}$:

$$
\mathcal{U}^{-}=\xi \pi f \sigma_{i} \mu_{i}^{2} \mathbf{K}^{t} \cdot\left[\begin{array}{c}
\left\langle H_{x}\right\rangle_{i}^{2} \\
\left\langle H_{y}\right\rangle_{i}^{2} \\
\left\langle H_{z}\right\rangle_{i}^{2}
\end{array}\right],
$$




$$
\mathcal{U}^{+}=\xi \pi f \sigma_{i} \mu_{i}^{2} \mathbf{K}^{t} \cdot\left[\begin{array}{c}
\left\langle H_{x}^{2}\right\rangle_{i} \\
\left\langle H_{y}^{2}\right\rangle_{i} \\
\left\langle H_{z}^{2}\right\rangle_{i}
\end{array}\right] .
$$

The shape factor $\mathbf{K}$ can also be obtained for other inclusion geometry such as square (2D) or cube (3D). This will be useful to describe SMCs with high volume fraction since the circular (2D) and spherical (3D) geometry of Fig. 1 cannot exceed filling factors of $78.54 \%$ and $52.36 \%$, respectively.

For the 2D case (square) with perpendicular magnetic field, the shape factor has an analytical form ${ }^{16}$ and its value can be approximated as $9 / 128 .{ }^{15}$ When the magnetic field is applied in plane, the EC loss density has the same expression as for a laminated steel, ${ }^{20}$ and the shape factor is $1 / 6$. Therefore, the geometry coefficient (containing the shape factor and the geometrical dimensions) can be written

$$
\mathbf{K}^{t}=\left[\frac{1}{6}, \frac{1}{6}, \frac{9}{128}\right] \pi L_{i}^{2},
$$

where $L_{i}$ is the size of the square. The two EC loss estimates $\mathcal{U}^{-}$and $\mathcal{U}^{+}$are then obtained for this microstructure (square cross-section cylinder) by introducing (9) into (7) and (8).

For the cube microstructure, it can be considered as square section in the three directions. The geometry coefficient is then written as

$$
\mathbf{K}^{t}=\left[\frac{9}{128}, \frac{9}{128}, \frac{9}{128}\right] \pi L_{i}^{2},
$$

where $L_{i}$ is the size of the cube. The two corresponding EC loss estimates $\mathcal{U}^{-}$and $\mathcal{U}^{+}$are similarly defined by introducing (10) into (7) and (8).

In Section III, it is proven that the exact value $\mathcal{U}$ of EC loss density lies between the two estimates $\mathcal{U}^{-}$and $\mathcal{U}^{+}$for a square lattice of cylinders or a cubic lattice of spheres.

\section{BOUNDS ON EC LOSSES}

Under the low frequency assumption, the magnetic field distribution can be deduced similar to magnetostatics conditions. The bound on EC loss density is discussed separately for SMCs with cylinder and sphere microstructure.

\section{A. Cylinder microstructure}

The case of cylinder microstructure can be simplified into a 2D problem. Magnetic field can be decomposed into two parts. One is the tangent component (in-plane part); the other is the normal component. The case of magnetic field applied in the normal direction (along the $z$-axis) has been discussed thoroughly ${ }^{15,16}$ since the magnetic field is uniform over the whole domain at low frequency

$$
H_{z}(\mathbf{x})=H_{z},
$$

where $\mathbf{x}$ is the position vector.

Since the magnetic field in the inclusion is uniform, (5) still holds

$$
\mathcal{U}_{z}=\frac{1}{4} \xi \pi^{2} R^{2} f \sigma_{i} \mu_{i}^{2} H_{z}^{2}
$$

The effective magnetic permeability can also be exactly determined by the upper Wiener bound ${ }^{21}$

$$
\mu_{z}=(1-\xi) \mu_{1}+\xi \mu_{i} .
$$

Consider now only the in-plane magnetic field loading, as indicated in Fig. 2. The problem is solved in polar coordinates $(r, \theta)$ and Cartesian coordinates $(x, y)$.

For a square lattice of circular inclusions excited by an average magnetic field $\overline{\mathbf{H}}$ (with $\overline{H_{z}}=0$ ) over the cell (and denoting $H=\|\overline{\mathbf{H}}\|$ ), the general solution for the magnetic scalar potential $\Phi$ (with $\mathbf{H}=-\nabla \Phi$ ) in the inclusion can be determined from the following equation in polar coordinates $^{22}$

$$
\begin{aligned}
\Phi_{i}(r, \theta)= & H R \sum_{n=0}^{\infty}\left(A_{n} \cos [(2 n+1) \theta]\right. \\
& \left.+B_{n} \sin [(2 n+1) \theta]\right)\left(\frac{r}{R}\right)^{2 n+1},
\end{aligned}
$$

where $A_{n}, B_{n}$ are real dimensionless coefficients depending on the average magnetic field direction, constituent properties, and volume fraction of the inclusion. The magnetic field in the inclusion in polar coordinates is then

$$
\begin{aligned}
\mathbf{H}_{i}(r, \theta)= & -H \sum_{n=0}^{\infty}\left(A_{n} \cos [(2 n+1) \theta]\right. \\
& \left.+B_{n} \sin [(2 n+1) \theta]\right)(2 n+1)\left(\frac{r}{R}\right)^{2 n} \vec{u}_{r} \\
& +H \sum_{n=0}^{\infty}\left(A_{n} \sin [(2 n+1) \theta]\right. \\
& \left.-B_{n} \cos [(2 n+1) \theta]\right)(2 n+1)\left(\frac{r}{R}\right)^{2 n} \vec{u}_{\theta},
\end{aligned}
$$

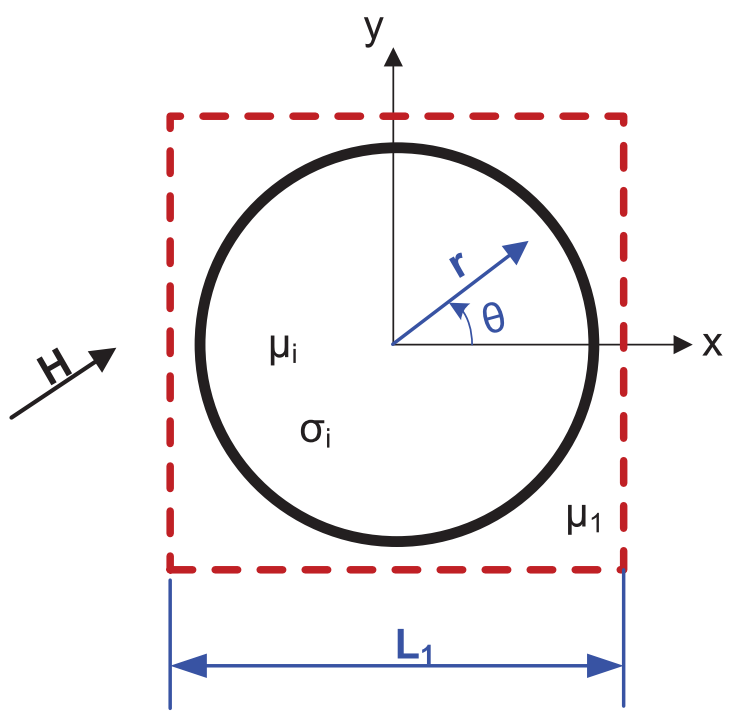

FIG. 2. Sketch of SMCs in a 2D problem with in-plane magnetic field loading. 
where $\vec{u}_{r}$ and $\vec{u}_{\theta}$ indicate unit vectors in polar coordinates. The induced electric field in the inclusion can be determined using (3)

$$
\begin{aligned}
E_{z}(r, \theta)= & j \omega \mu_{i} H R \sum_{n=0}^{\infty}\left(A_{n} \sin [(2 n+1) \theta]\right. \\
& \left.-B_{n} \cos [(2 n+1) \theta]\right)\left(\frac{r}{R}\right)^{2 n+1} .
\end{aligned}
$$

The in-plane electric field is zero (with $E_{r}=E_{\theta}=0$ ).

Applying the definition of EC loss density (1), the exact value can be determined (while considering no loss in the dielectric matrix surrounding the inclusion)

$$
\mathcal{U}_{x, y}=\frac{1}{2} \xi \pi^{2} R^{2} f \sigma_{i} \mu_{i}^{2} H^{2} \sum_{n=0}^{\infty} \frac{1}{n+1}\left(A_{n}^{2}+B_{n}^{2}\right) .
$$

Now, let consider the two estimates for EC losses given by (7) and (8). Using (15), the average field and second order moment in the inclusion are, respectively

$$
\left\{\begin{array}{l}
\langle\mathbf{H}\rangle_{i}=-H\left(A_{0} \vec{u}_{x}+B_{0} \vec{u}_{y}\right) \\
\left\langle\mathbf{H}^{2}\right\rangle_{i}=H^{2} \sum_{n=0}^{\infty}(2 n+1)\left(A_{n}^{2}+B_{n}^{2}\right),
\end{array}\right.
$$

which lead to the following EC loss density estimates:

$$
\left\{\begin{array}{l}
\mathcal{U}_{x, y}^{-}=\frac{1}{2} \xi \pi^{2} R^{2} f \sigma_{i} \mu_{i}^{2} H^{2}\left(A_{0}^{2}+B_{0}^{2}\right) \\
\mathcal{U}_{x, y}^{+}=\frac{1}{2} \xi \pi^{2} R^{2} f \sigma_{i} \mu_{i}^{2} H^{2} \sum_{n=0}^{\infty}(2 n+1)\left(A_{n}^{2}+B_{n}^{2}\right) .
\end{array}\right.
$$

Comparing (17) to (19), the two estimates clearly define bounds on the EC loss density

$$
\mathcal{U}_{x, y}^{-} \leq \mathcal{U}_{x, y} \leq \mathcal{U}_{x, y}^{+}
$$

When the magnetic field in the inclusion is uniform, $\mathbf{H}_{i}(r, \theta)$ in (15) should be $r$-independent, which gives

$$
A_{n}=0, \quad B_{n}=0 \quad \forall n \in \mathbb{N}^{+} .
$$

Substituting (21) into (17) to (19) lead to the equality in (20). This is the case of dilute approximation.

The three EC loss density estimates are also identical for the component of the magnetic field in the $z$-direction

$$
\mathcal{U}_{z}^{-}=\mathcal{U}_{z}=\mathcal{U}_{z}^{+} .
$$

Combining the cases of perpendicular field and in-plane field, the following generic bounding equation holds for SMCs with cylinder microstructure:

$$
\mathcal{U}^{-} \leq \mathcal{U} \leq \mathcal{U}^{+}
$$

\section{B. Sphere microstructure}

For a cubic lattice of spherical inclusions, we consider first a magnetic field applied along the $z$-direction. The general case can be obtained using symmetries (see Appendix A for detailed proof).

In spherical coordinates $(r, \theta, \phi)$, shown in Fig. 3, the magnetic scalar potential $\Phi$ (with $\mathbf{H}=-\nabla \Phi)$ can be solved by the Laplace equation $\nabla^{2} \Phi=0$. The general solution has the form ${ }^{23}$

$$
\Phi(r, \theta, \phi)=\sum_{l=0}^{\infty} \sum_{m=-l}^{l}\left[A_{l m} r^{l}+B_{l m} r^{-(l+1)}\right] Y_{l m}(\theta, \phi),
$$

where $A_{l m}$ and $B_{l m}$ are coefficients to be determined by the boundary conditions. $Y_{l m}(\theta, \phi)$ is the normalized spherical harmonic function

$$
Y_{l m}(\theta, \phi)=\sqrt{\frac{2 l+1}{4 \pi} \frac{(l-m) !}{(l+m) !}} P_{l}^{m}(\cos \theta) e^{j m \phi},
$$

with $P_{l}^{m}(\cos \theta)$ the Legendre polynomials.

If it concerns only the sphere (the inclusion), $B_{l m}=0$. In addition, $l$ is restricted to odd integers $(l=1,3,5, \ldots)$ and $m$ to non-negative integer multiples of $4(m=0,4$, $8, \ldots) .{ }^{24,25}$ Denote $H=\|\overrightarrow{\mathbf{H}}\|$. The potential equation can be rewritten as

$$
\Phi_{i}(r, \theta, \phi)=H R \sum_{n=0}^{\infty} \sum_{m=0}^{\left\lfloor\frac{2 n+1}{4}\right\rfloor} C_{2 n+1,4 m}\left(\frac{r}{R}\right)^{2 n+1} Y_{2 n+1,4 m}(\theta, \phi),
$$

with $\lfloor\cdot\rfloor$ representing the floor operator. $C_{n, m}$ are real dimensionless coefficients.

Apply

$$
\mathbf{H}=-\nabla \Phi=-\frac{\partial \Phi}{\partial r} \vec{u}_{r}-\frac{1}{r} \frac{\partial \Phi}{\partial \theta} \vec{u}_{\theta}-\frac{1}{r \sin \theta} \frac{\partial \Phi}{\partial \phi} \vec{u}_{\phi},
$$

where $\vec{u}_{r}, \vec{u}_{\theta}$, and $\vec{u}_{\phi}$ are unit vectors in spherical coordinates. The magnetic field distribution in the inclusion can then be obtained.

Given the magnetic field distribution in the sphere, the induced electric field can be analytically deduced. Numerical calculations show that azimuthal induced electric field is negligible. This is also discussed in Ref. 26. Finally, the expression for $E_{\phi}$ is

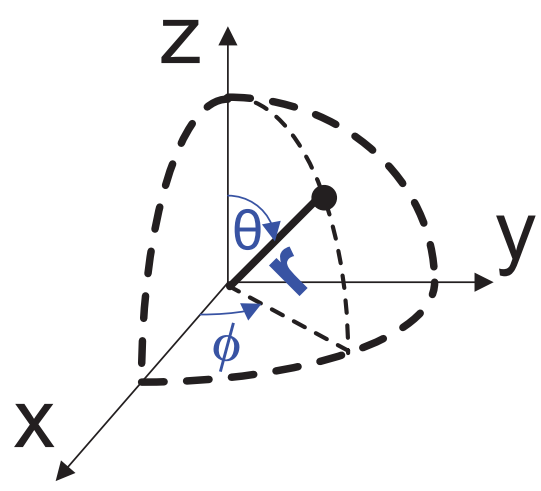

FIG. 3. Spherical coordinates $(r, \theta, \phi)$. 
$E_{\phi}(r, \theta, \phi)=-j \omega \mu H R \sum_{n=0}^{\infty} \frac{C_{2 n+1,0}}{2 n+2}\left(\frac{r}{R}\right)^{2 n+1} \frac{\partial}{\partial \theta} Y_{2 n+1,0}(\theta, \phi)$,

with $E_{r}=E_{\theta}=0$.

Substituting (28) into the definition (1) leads to the exact EC loss density formula

$$
\mathcal{U}_{z}=\frac{3}{4} \xi \pi R^{2} f \sigma_{i} \mu_{i}^{2} H^{2} \sum_{n=0}^{\infty} \frac{2 n+1}{(n+1)(4 n+5)} C_{2 n+1,0}^{2}
$$

The average magnetic field $\langle\mathbf{H}\rangle_{i}$ and the second order moment $\left\langle\mathbf{H}^{2}\right\rangle_{i}$ in the sphere are obtained from (27)

$$
\left\{\begin{aligned}
\langle\mathbf{H}\rangle_{i} & =-\sqrt{\frac{3}{4 \pi}} H C_{1,0} \\
\left\langle\mathbf{H}^{2}\right\rangle_{i} & =\frac{3}{4 \pi} H^{2} \sum_{n=0}^{\infty}(2 n+1) C_{2 n+1,0}^{2} .
\end{aligned}\right.
$$

Substituting them into (7) and (8) leads to

$$
\left\{\begin{array}{l}
\mathcal{U}_{z}^{-}=\frac{3}{20} \xi \pi R^{2} f \sigma_{i} \mu_{i}^{2} H^{2} C_{1,0}^{2} \\
\mathcal{U}_{z}^{+}=\frac{3}{20} \xi \pi R^{2} f \sigma_{i} \mu_{i}^{2} H^{2} \sum_{n=0}^{\infty}(2 n+1) C_{2 n+1,0}^{2} .
\end{array}\right.
$$

Again, comparing to (29), it can be concluded that

$$
\mathcal{U}_{z}^{-} \leq \mathcal{U}_{z} \leq \mathcal{U}_{z}^{+}
$$

Due to symmetry, the inequality also holds when the magnetic field is applied along the $x$ - or $y$-axis separately. The corresponding EC loss density is $\mathcal{U}_{x}$ or $\mathcal{U}_{y}$, respectively. As discussed in Appendix A, the final EC loss density can be directly obtained by adding the three terms: $\mathcal{U}=\mathcal{U}_{x}+\mathcal{U}_{y}+\mathcal{U}_{z}$. The same addition rule applies for the first and second order moments of the magnetic field. Therefore, for a magnetic field in the arbitrary direction, we have

$$
\mathcal{U}^{-} \leq \mathcal{U} \leq \mathcal{U}^{+}
$$

It is now proven that the EC loss density estimates obtained by using the average magnetic field or second order moment in the inclusions [(7) and (8)] are lower and upper bounds for the EC loss density in the case of a cubic lattice of spheres.

\section{Discussion about the bounds}

When the magnetic field in the inclusions is uniform, both bounds are equal to the exact EC loss value, justifying the equality case in Eqs. (23) and (33).

But when the magnetic field is non-uniform, the EC loss value strictly stands between the two bounds. Hence, the bounds are not optimal: there is no known configuration where the EC loss attains a bound value.

Nevertheless, EC loss value can be exactly determined only by knowing the complete distribution of magnetic field in the inclusions. Extracting such information is usually complex and expensive (e.g., use of full-field approach such as FEM).

The proposed approach in this paper is to use homogenization techniques in order to build these two simple closedform expressions (based on average and second-order moment of magnetic field) for EC losses estimate. The real EC loss value is bounded by these two closed-form estimates (arithmetic proof).

In Sections IV and V, the use of such bounds will be discussed and criticized.

\section{Extension to more generic microstructures}

Consider a biphasic matrix-inclusion composite with linear and isotropic constituent properties. It is excited by a macroscopic flux density $\|\overline{\mathbf{B}}\|=B_{0}$. Given the effective permeability $\tilde{\mu}$, the average magnetic field $\langle\mathbf{H}\rangle_{i}$ and the second order moment of magnetic field $\left\langle\mathbf{H}^{2}\right\rangle_{i}$ in the inclusions can be retrieved ${ }^{19}$

$$
\langle\mathbf{H}\rangle_{i}=\frac{\tilde{\mu}-\mu_{1}}{\xi\left(\mu_{i}-\mu_{1}\right)} \overline{\mathbf{H}}=\frac{\tilde{\mu}-\mu_{1}}{\xi \tilde{\mu}\left(\mu_{i}-\mu_{1}\right)} \overline{\mathbf{B}}
$$

and

$$
\left\langle\mathbf{H}^{2}\right\rangle_{i}=\frac{1}{\xi} \frac{\partial \tilde{\mu}}{\partial \mu_{i}} \overline{\mathbf{H}}^{2}=\frac{1}{\xi \tilde{\mu}^{2}} \frac{\partial \tilde{\mu}}{\partial \mu_{i}} \overline{\mathbf{B}}^{2}
$$

where $\mu_{1}$ is the permeability of the dielectric matrix.

For an arbitrary composite, if enough geometry information, noted $\Xi$, is given, the effective permeability can be obtained as a function of the geometry information and the properties of the constituents: $\tilde{\mu}\left(\mu_{1}, \mu_{i}, \Xi\right)$. The shape factor $K(\Xi)$ can be obtained from (6) or as in Ref. 16, considering simplified basic shapes for the inclusions. Substituting $\tilde{\mu}\left(\mu_{1}, \mu_{i}, \Xi\right)$ into (34) and (35), the first and second order moment of magnetic field can be obtained, which leads to the lower and upper EC loss density bounds $\left(\mathcal{U}^{+}\right.$and $\left.\mathcal{U}^{-}\right)$, respectively. The bounds as a function of volume fraction of inclusions $\xi$ are plotted in Fig. 4 in a schematic way. The EC loss density values are normalized by the value at $\xi=1$.

At very low or very high volume fraction, the magnetic field in the inclusion can be considered uniform, so that,

$$
\left\langle\mathbf{H}^{2}\right\rangle_{i} \approx\langle\mathbf{H}\rangle_{i}^{2}
$$

At the middle range of volume fraction, the magnetic field in the inclusion is distorted, so that

$$
\left.\left\langle\mathbf{H}^{2}\right\rangle_{i}\right\rangle\langle\mathbf{H}\rangle_{i}^{2}
$$

Therefore, there is a gap between the two bounds.

The EC loss density bounds depend on the permeability contrast, as shown in Fig. 5.

At low permeability contrast, the magnetic field in the inclusion can be considered uniform, so that the two bounds have the same value. As the permeability contrast increases, the magnetic field becomes distorted. The two bounds separate. When the permeability contrast is big enough, the 


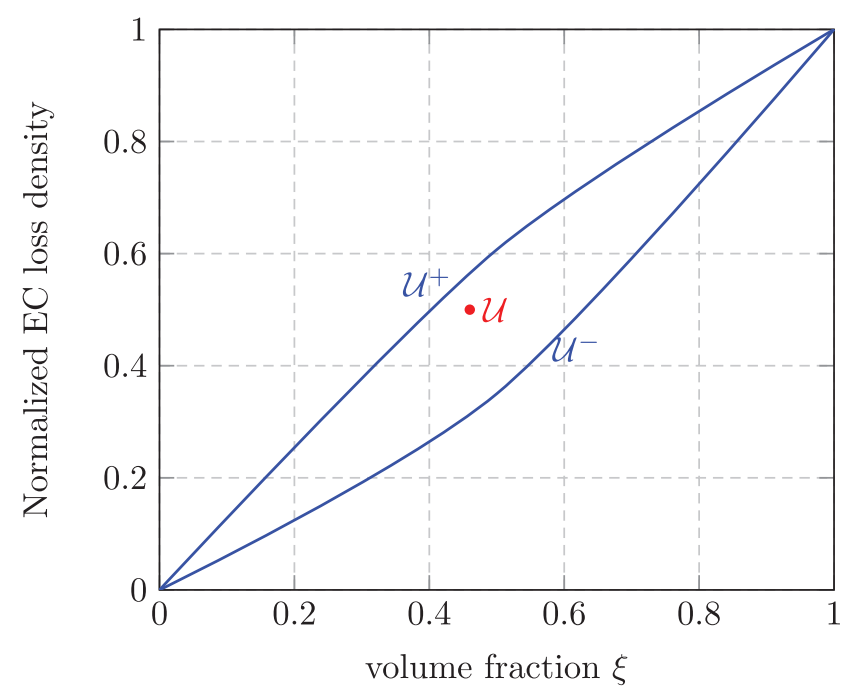

FIG. 4. A schematic plot of EC loss density bounds as a function of volume fraction of the inclusion.

change of the field distribution brought by the increase in the permeability is negligible. Therefore, the space between the two bounds remains unchanged when the permeability contrast is high enough.

The EC loss density bounds also vary as a function of frequency, as shown in Fig. 6.

At low frequency, the contribution of the induced magnetic field is neglected. The field distribution is independent of frequency. The two bounds are proportional to frequency just as the EC loss density. Therefore, the gap between the two bounds is also proportional to frequency.

To conclude, if the effective permeability $\tilde{\mu}\left(\mu_{1}, \mu_{i}, \Xi\right)$ and the shape factor $K(\Xi)$ are correctly determined, the lower and upper bounds for EC loss density can be obtained. Nevertheless, in most cases, the microstructure information is not sufficiently provided. Usually, with limited geometry information, the effective permeability can only be estimated and the form of the inclusions can be approximated into basic shapes. If an estimate for effective permeability is

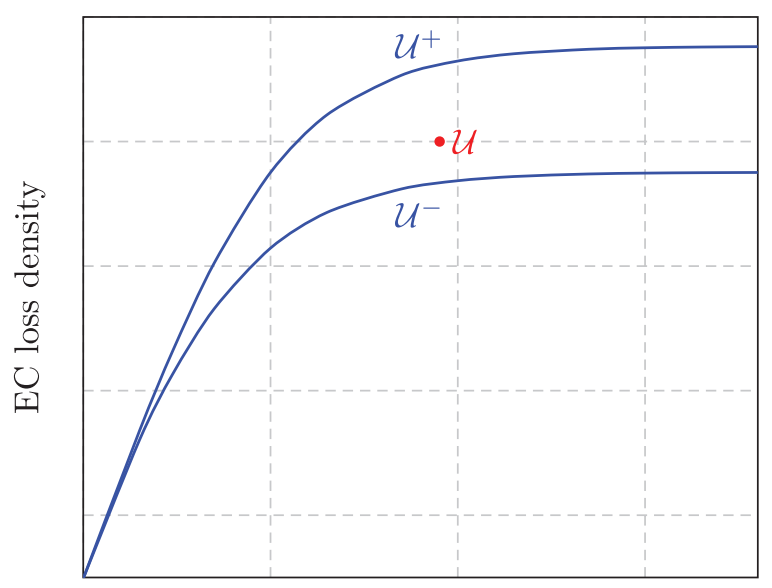

Permeability contrast, $\mu_{i} / \mu_{1}$

FIG. 5. A schematic plot of EC loss density bounds as a function of permeability contrast.

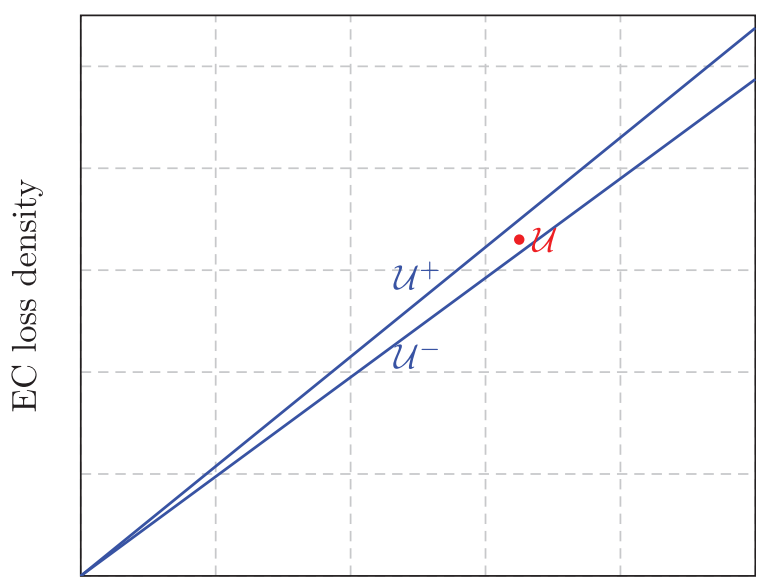

Frequency

FIG. 6. A schematic plot of EC loss density bounds as a function of frequency.

used, then, $\mathcal{U}^{-}$and $\mathcal{U}^{+}$cannot rigorously be considered as bounds. They will be referred to later as lower and upper estimates.

\section{EC LOSS DENSITY ESTIMATES}

The lower and upper estimates depend greatly on the determination of the effective permeability of the composite. In most cases, the effective permeability cannot be exactly obtained, and its value has to be approximated.

Maxwell Garnett (MG) estimate is a simple and popular approach for the determination of the effective properties of composites. The effective permeability has the form ${ }^{13,27}$

$$
\tilde{\mu}_{\mathrm{MG}}=\mu_{1}+\frac{\xi \mu_{1}\left(\mu_{i}-\mu_{1}\right)}{\mu_{1}+N(1-\xi)\left(\mu_{i}-\mu_{1}\right)},
$$

where $N$ is a depolarization coefficient: $N=\frac{1}{3}$ for sphere microstructure and $N=\frac{1}{2}$ for cylinder microstructure. ${ }^{13}$ Substituting (38) into (34) and (35) leads to

$$
\left\langle\mathbf{H}^{2}\right\rangle_{i}=\langle\mathbf{H}\rangle_{i}^{2} .
$$

Therefore, there is only one EC loss density estimate, denoted as $\mathcal{U}_{\mathrm{MG}}$. MG estimate provides a satisfying approximation for dilute SMCs with circular or spherical inclusions. An alternative effective permeability estimate can be obtained using series expansions, ${ }^{22,25}$ which can attain high precision by calculating high order terms even for high volume fractions $\xi$. Therefore, (34) and (35) can be determined and provide EC loss density estimates by substitution into (7) and (8).

In the following, EC loss density estimates for periodic SMCs with circular inclusion or spherical inclusions are obtained with series expansions. For SMCs with high volume fraction of inclusions (greater than $78.54 \%$ for $2 \mathrm{D}$ case, or greater than $52.36 \%$ for $3 \mathrm{D}$ case), the inclusions cannot be considered circular or spherical without overlapping. The inclusions are then taken as squares or cubes. The estimates are compared with numerical simulations. Finite Element 
TABLE I. Material parameters used in the calculations.

\begin{tabular}{lccc}
\hline \hline & $\begin{array}{c}\text { Conductivity } \\
(\mathrm{S} / \mathrm{m})\end{array}$ & $\begin{array}{c}\text { Relative } \\
\text { permeability }\end{array}$ & $\begin{array}{c}\text { Relative } \\
\text { permittivity }\end{array}$ \\
\hline Iron & $1.12 \times 10^{7}$ & 4000 & 1 \\
Epoxy & $1.7 \times 10^{-13}$ & 1 & 9 \\
\hline \hline
\end{tabular}

Method (FEM) calculations have been performed on a unit cell of SMCs as described in Fig. 1 for different volume fractions at different frequencies. EC loss densities $\mathcal{U}_{\text {FEM }}$ are post-processed from FEM calculations and are considered as reference values.

The cell size $L_{1}$ (lattice length) is fixed to $50 \mu \mathrm{m}$. The average flux density over a cell $B_{0}$ is fixed to $1 \mathrm{~T}$. The material properties used for the constituents are given in Table I.

\section{A. Cylinder microstructure}

The effective permeability $\tilde{\mu}_{\mathrm{MG} 2}$ is determined by $\mathrm{MG}$ estimate using (38). The subscript 2 refers to the $2 \mathrm{D}$ Maxwell-Garnett estimate $\left[N=1 / 2\right.$ in (38)]. $\tilde{\mu}_{\mathrm{MG} 2}$ is used to obtain EC loss density estimate, $\mathcal{U}_{\mathrm{MG}}$.

On the other hand, series expansion approaches provide more accurate approximations of effective properties than the MG estimates. The effective permeability is obtained using series expansion with the Godin's formula (B1) recalled in Appendix B.

In the following results, $\mathcal{U}_{\mathrm{G}}^{-}$and $\mathcal{U}_{\mathrm{G}}^{+}$are determined by using the effective permeability given by Godin's formula (B1). The effective permeability is calculated to the order $\xi^{12}$ in (B2).

\section{EC loss density as a function of the filling factor}

Numerical EC loss density $\mathcal{U}_{\text {FEM }}$ and analytical bounds $\mathcal{U}_{\mathrm{G}}^{-}$and $\mathcal{U}_{\mathrm{G}}^{+}$as a function of the filling factor $\xi$ of cylinders are plotted in Fig. 7. The superscript "cir" indicates the results for cylinders with circular cross section, while the "squ" for cylinders with square cross section.

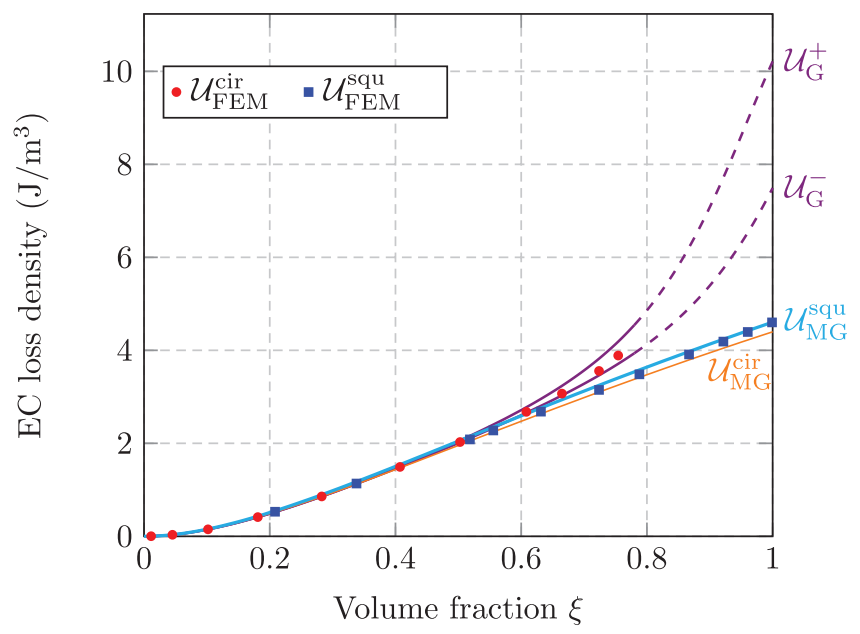

FIG. 7. EC loss density as a function of the inclusion filling factor. For all calculations: $f=100 \mathrm{~Hz}$, lattice size $L_{1}=50 \mu \mathrm{m}, \mu_{i}=4000 \mu_{0}, \sigma_{i}=1.12$ $\times 10^{7} \mathrm{~S} / \mathrm{m}$, and average flux density $B_{0}=1 \mathrm{~T}$.
As expected EC losses increase when the volume fraction of the conductive inclusions becomes higher. For squareshaped inclusions of size $L_{i}$, the EC loss density estimate is

$$
\begin{aligned}
\mathcal{U}_{\mathrm{MG}}^{\mathrm{squ}} & =\frac{1}{6} \xi \pi^{2} L_{i}^{2} f \sigma_{i} \mu_{i}^{2}\langle\mathbf{H}\rangle_{i}^{2} \\
& =\frac{1}{6} f \sigma_{i}\left(\frac{2 \pi L_{1} B_{0} \mu_{i} \xi}{\mu_{1}+\mu_{i}+\left(\mu_{i}-\mu_{1}\right) \xi}\right)^{2} .
\end{aligned}
$$

$\mathcal{U}_{\mathrm{MG}}^{\mathrm{squ}}$ is a monotonically increasing function of $\xi$.

For the case of circular inclusions, given $\mu_{i}>\mu_{1}$, (B3) satisfies $0<\alpha<1$, which results in $\tilde{\mu}_{\mathrm{G}}$ in (B1) being a monotonically increasing function of $\xi$ (see Appendix B). The EC loss density estimate from the average magnetic field approach has the form

$$
\begin{aligned}
\mathcal{U}_{\mathrm{G}}^{-} & =\frac{1}{2} \xi \pi^{2} R^{2} f \sigma_{i} \mu_{i}^{2}\langle\mathbf{H}\rangle_{i}^{2} \\
& =\frac{1}{2} \pi L_{1}^{2} f \sigma_{i} B_{0}^{2}\left(\frac{1}{1-\mu_{1} / \mu_{i}}\right)^{2}\left(1-\frac{\mu_{1}}{\tilde{\mu}}\right)^{2} .
\end{aligned}
$$

Therefore, $\mathcal{U}^{-}$increases monotonically with the volume fraction $\xi$.

At low volume fraction, all approximations $\left(\mathcal{U}_{\mathrm{G}}^{-}, \mathcal{U}_{\mathrm{G}}^{+}\right.$, and $\left.\mathcal{U}_{\mathrm{MG}}\right)$ agree very well with the FEM results. The reason is that, at low volume fraction, the magnetic field in the inclusion is almost uniform (dilute approximation). The uniformity of the magnetic field provides the condition of equality in (20). Also for a given low volume fraction, the numerical results and estimates are the same no matter the cross section is a circle or a square.

For a middle range volume fraction $(0.5<\xi<0.75)$, the Godin approximations $\left(\mathcal{U}_{\mathrm{G}}^{-}\right.$and $\left.\mathcal{U}_{\mathrm{G}}^{+}\right)$coincide with FEM results for circle-shaped inclusions. The space between the two estimates is getting bigger as the volume fraction increases. This is due to the increasing distortion of the magnetic field within the inclusions when the volume fraction is getting higher. In such distorted cases, MG is not valid anymore. MG estimate can be used with satisfactory accuracy when the field in the inclusion is reasonably uniform. This is the case for very low volume fraction (dilute case) but also for high volume fraction (such as high volume fraction of square-shaped inclusions).

At high volume fraction $(\xi>0.79)$, inclusion shapes cannot be circular without overlapping. But square inclusions can reach these high volume fractions. MG estimate still provides reliable approximations of EC loss density. It is also remarkable that, using $\mathrm{MG}$ estimate in $2 \mathrm{D}$, same levels of EC losses are obtained for circular and square inclusions. It can be seen from the respective definitions of $\mathbf{K}$ in both cases [(6) and (9)] that $\mathcal{U}_{\mathrm{MG}}^{\mathrm{squ}} / \mathcal{U}_{\mathrm{MG}}^{\mathrm{cir}}=\pi / 3$.

As a summary, for cylinder microstructure with circular cross-section, Godin's formula is used to obtain lower and upper estimates of the EC losses. For square-shaped crosssection, MG estimate is a good choice to obtain an estimate of EC losses. It is expected that for high volume fraction inclusion-matrix type composites, the MG estimate is a good approximation for the effective permeability as long as the distribution of the inclusions can be considered random. 


\section{EC loss density as a function of permeability contrast}

In this section, EC losses are examined with respect to permeability contrast. MG estimate is only plotted for SMCs with square-shaped inclusions, the case of circular inclusions being very similar. Permeability contrast is defined as $c_{\mu}$ $=\mu_{i} / \mu_{1}$. Since the matrix material is nonmagnetic, $\mu_{1}=\mu_{0}$. Results are plotted in Fig. 8.

For all the calculations, the filling factor of inclusions is set to $\xi=72.38 \%$. For circular inclusions, the radius is $R=24 \mu \mathrm{m}$, and for square-shaped inclusions, the size is $L_{i}$ $=42.5 \mu \mathrm{m}$. When the permeability contrast is high (greater than a few hundred), EC loss density values saturate. This can be understood from the calculation of the effective permeability (see Appendix B). When the contrast is very high $\left(\mu_{i} \gg \mu_{1}\right)$, the effective permeability $\tilde{\mu}_{\mathrm{G}}$ becomes independent of $\mu_{i}$. For the case of circular inclusion, the lower and upper estimates have the forms

$$
\begin{aligned}
\mathcal{U}_{\mathrm{G}}^{-} & =\frac{1}{2} \xi \pi^{2} R^{2} f \sigma_{i} B_{0}^{2}\left(\frac{1}{1-\mu_{1} / \mu_{i}}\right)^{2}\left(\frac{2}{1+\lambda \xi}\right)^{2} \lambda^{2} \\
& \approx \frac{1}{2} \xi \pi^{2} R^{2} f \sigma_{i} B_{0}^{2}\left(\frac{2}{1+\lambda \xi}\right)^{2} \lambda^{2} \quad \text { as } \quad \mu_{i} \gg \mu_{1}
\end{aligned}
$$

and

$$
\begin{aligned}
\mathcal{U}_{\mathrm{G}}^{+}= & \frac{1}{2} \xi \pi^{2} R^{2} f \sigma_{i} B_{0}^{2}\left(\frac{1}{1+\mu_{1} / \mu_{i}}\right)^{2} \\
& \times\left(\frac{2}{1+\lambda \xi}\right)^{2}\left(1+0.9175 \alpha^{2} \xi^{4}+\cdots\right) \\
\approx & \frac{1}{2} \xi \pi^{2} R^{2} f \sigma_{i} B_{0}^{2}\left(\frac{2}{1+\lambda \xi}\right)^{2} \\
& \times\left(1+0.9175 \alpha^{2} \xi^{4}+\cdots\right) \text { as } \mu_{i} \gg \mu_{1},
\end{aligned}
$$

so that $\mathcal{U}^{-}$and $\mathcal{U}^{+}$are also $c_{\mu}$-independent and the inequality $\mathcal{U}^{-}<\mathcal{U}^{+}$holds.

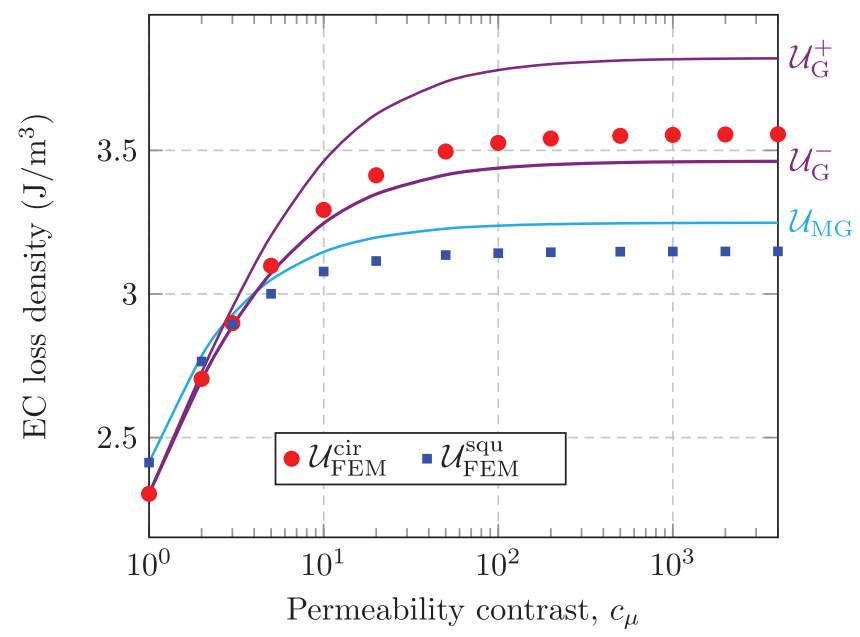

FIG. 8. EC loss density as a function of the permeability contrast. Parameters: $f=100 \mathrm{~Hz}$, lattice size $L_{1}=50 \mu \mathrm{m}, \xi=72.38 \%, \mu_{1}=\mu_{0}, \sigma_{i}=1.12$ $\times 10^{7} \mathrm{~S} / \mathrm{m}$, and average flux density $B_{0}=1 \mathrm{~T}$.
For the case of square-shaped inclusions, the effective permeability is determined by MG estimate and the EC loss density formula writes

$$
\begin{aligned}
\mathcal{U}_{\mathrm{MG}} & =\frac{1}{6} \pi^{2} L_{1}^{2} f \sigma_{i} B_{0}^{2}\left(\frac{2 \xi c_{\mu}}{1-\xi+(1+\xi) c_{\mu}}\right)^{2} \\
& \approx \frac{1}{6} \pi^{2} L_{1}^{2} f \sigma_{i} B_{0}^{2}\left(\frac{2 \xi}{1+\xi}\right)^{2} \quad \text { as } \quad c_{\mu} \gg 1 .
\end{aligned}
$$

For low contrast, the differences between estimates and the reference values are very small. This is expected since the magnetic field is almost uniform in the inclusions for low permeability contrast even for high filling factors. Because the volume fraction is fixed, when $c_{\mu} \gg 1, \tilde{\mu}$ approaches a constant [this can be shown either from series expansion (B1) or from MG estimate (38)]. Thus, the variation of the magnetic field distribution can be neglected. Finally, the discrepancy between the MG approach and the FEM results remains constant as $c_{\mu}$ increases (when $c_{\mu}>100$ in Fig. 8). This discrepancy is attributed to the approximation on the effective permeability estimate.

\section{B. Sphere microstructure}

Similar to the case of cylinder microstructure, for cubic inclusions, $\tilde{\mu}_{\mathrm{MG} 3}$ is used to obtain EC loss density estimate, $\mathcal{U}_{\text {MG }}$. The subscript 3 refers to the 3D Maxwell-Garnett estimate $[N=1 / 3$ in (38)]. For spherical inclusions, the effective permeability is obtained using series expansion with Lam's formula (B4) (see Appendix B). In the following results, $\mathcal{U}_{\text {Lam }}^{-}$and $\mathcal{U}_{\text {Lam }}^{+}$are determined using the effective permeability given by Lam's formula (B4). The effective permeability is calculated to the order $\xi^{6}$ in (B5).

\section{EC loss density as a function of the filling factor}

EC loss density as a function of the filling factor $\xi$ of inclusions is plotted in Fig. 9. The superscript "sph" indicates the case of SMCs with spherical inclusions, and "cub" stands for cubic.

Similar conclusions can be drawn for SMCs with spherical inclusions (3D) as for SMCs with cylindrical inclusions (2D). At low volume fraction (dilute assumption), MG estimate provides a reliable approximation for EC loss density. That is because the magnetic field can be considered as uniform within the inclusions. At middle volume fraction, when the inclusions are assumed spherical and the microstructure periodic, MG estimate is unacceptable, while the lower and upper estimates $\left(\mathcal{U}_{\text {Lam }}^{-}\right.$and $\left.\mathcal{U}_{\text {Lam }}^{+}\right)$ are valid. The difference between $\mathcal{U}_{\text {Lam }}^{-}$and $\mathcal{U}_{\text {Lam }}^{+}$increases with the volume fraction. For cubic-shaped inclusions, MG estimate provides a good approximation of EC loss density for all volume fractions.

Again similar to the 2D case, it can be concluded that for spherical microstructures, Lam's approach is the better option to obtain lower and upper estimates of the EC losses. For cubic-shaped inclusions, MG estimate is a good choice to obtain an estimate of EC losses. It is expected that for high volume fraction inclusion-matrix type composites, MG estimate is 


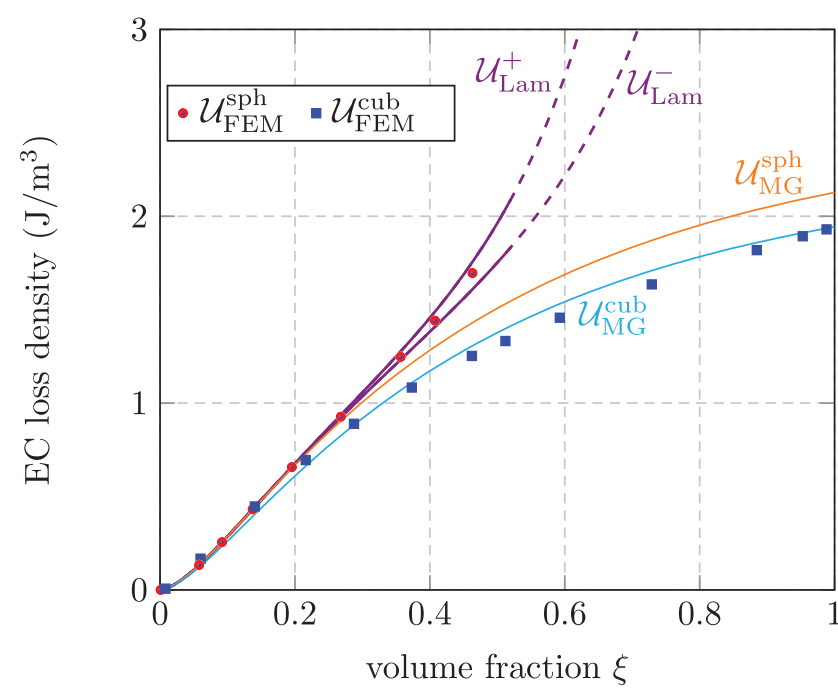

FIG. 9. EC loss density as a function of the filling factor of the inclusions. Parameters: $f=100 \mathrm{~Hz}$, lattice size $L_{1}=50 \mu \mathrm{m}, \mu_{i}=4000 \mu_{0}, \mu_{1}=\mu_{0}, \sigma_{i}$ $=1.12 \times 10^{7} \mathrm{~S} / \mathrm{m}$, and average flux density $B_{0}=1 \mathrm{~T}$.

a good approximation for the effective permeability as long as the distribution of the inclusions can be considered random. In the following discussion on the effect of permeability contrast, MG estimate is examined for SMCs with cubic inclusions. The case of spherical inclusions is very similar, and it can be shown that $\mathcal{U}_{\mathrm{MG}}^{\mathrm{cub}} / \mathcal{U}_{\mathrm{MG}}^{\mathrm{sph}}=5 / 32 \sqrt[3]{(9 \pi / 2)^{2}} \approx 0.91$.

\section{EC loss density as a function of permeability contrast}

EC loss density also depends on the permeability contrast, $c_{\mu}$. Figure 10 presents the EC loss density as a function of the permeability contrast for a filling factor set to $\xi$ $=46.32 \%$. For spherical inclusions, the radius is $R=24 \mu \mathrm{m}$, and for cubic inclusions, the size is $L_{i}=38.7 \mu \mathrm{m}$.

Again, similar conclusions as for the 2D case can be drawn for the $3 \mathrm{D}$ case. A similar formula can be obtained for $\mathcal{U}^{-}$which is shown to be $c_{\mu}$-independent at high permeability contrast

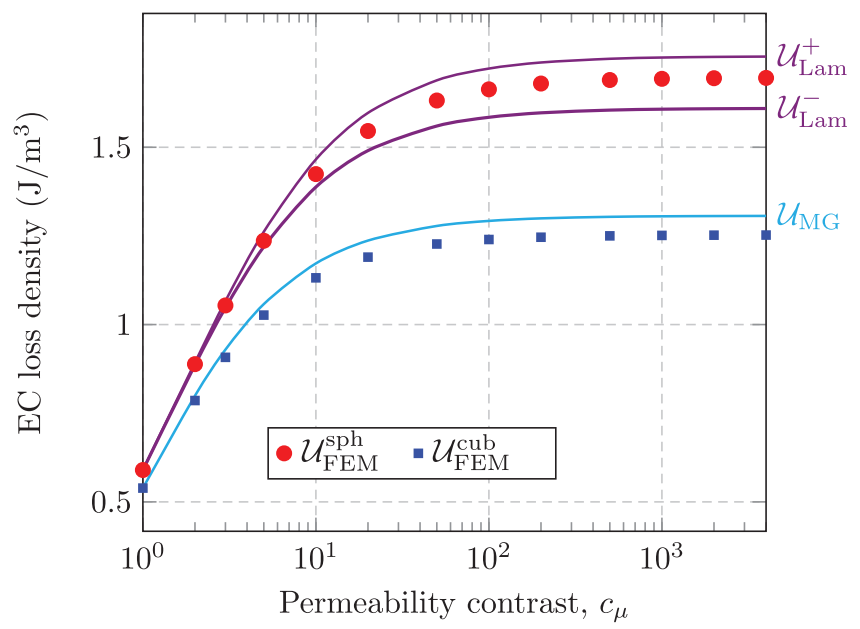

FIG. 10. EC loss density as a function of the permeability contrast. For all calculations: $f=100 \mathrm{~Hz}$, lattice size $L_{1}=50 \mu \mathrm{m}, \xi=46.32 \%, \mu_{1}=\mu_{0}, \sigma_{i}$ $=1.12 \times 10^{7} \mathrm{~S} / \mathrm{m}$, and average flux density $B_{0}=1 \mathrm{~T}$.

$$
\begin{aligned}
\mathcal{U}_{\text {Lam }}^{-} & =\frac{1}{5} \xi \pi^{2} R^{2} f \sigma_{i} B_{0}^{2}\left(\frac{1}{1-\mu_{1} / \mu_{i}}\right)^{2}\left(\frac{3}{\gamma+3 \xi}\right)^{2} \xi^{2} \\
& \approx \frac{1}{5 \xi} \pi^{2} R^{2} f \sigma_{i} B_{0}^{2}\left(\frac{3}{\gamma+3 \xi}\right)^{2} \text { as } \mu_{i} \gg \mu_{1} .
\end{aligned}
$$

\section{APPLICATION}

This section is dedicated to an illustration of the application of the modelling approach. A few guidelines for the practical application of the method are given first. A purely numerical example is then detailed in order to assess the validity of the proposed model. A comparison to experimental results from the literature is finally discussed.

\section{A. Application guidelines}

Depending on the level of knowledge on a given heterogeneous material (constituent properties, microstructure, etc.), the homogenization approach can be applied at different complexity levels:

1. If only the constituent properties are known, an homogenization model (Wiener, 2D or 3D Maxwell-Garnett, Bruggeman, Lam, Godin models) has to be chosen, ideally as a function of the type of microstructure ${ }^{28}$ so as to determine the effective permeability $\tilde{\mu}$. The average field in the inclusions and the second order moment can then be defined by (34) and (35), respectively. The shape factor $\mathbf{K}^{t}$ has to be calculated depending on the microstructure. Lower and upper estimates for the EC losses are then calculated using (7) and (8), respectively. For standard SMCs, MG estimate for the effective permeability and a cube shape for the definition of $\mathbf{K}^{t}$ (10) usually provide an accurate estimate of the EC loss density.

2. If in addition there is a possibility to know accurately the effective permeability (for instance by an experimental measurement or through a quasi-static FEM computation), then there is no need to use a homogenization model. The average field per phase can be obtained directly from (34), and an estimate for the EC losses using (7). Such an approach will avoid the possible inaccuracy related to the choice of a homogenization strategy, but will not provide lower and upper estimates since the second order moments cannot be defined.

3. An even more precise approach could be obtained if the microstructure is precisely known (for instance through a microscope image of the material). Then a quasistatic FEM computation can be performed on this microstructure, providing not only the effective permeability but also the average field in the inclusions and the second order moment of the field. Given $\mathbf{K}^{t}$ (for high volume fraction the cubic approximation will generally be sufficient), lower and upper estimates can be obtained using (7) and (8).

Of course a full dynamic FEM calculation can provide the values for EC losses, but this is usually the type of dynamic computations that we try to avoid. These computations will be performed in the following part, as a means of validation for the estimates obtained using the proposed methodology. 


\section{B. Numerical example}

In this section, the proposed estimates are tested against a more generic range of microstructures for SMCs. Due to the limitations for FEM computations, only 2D microstructures are considered, but the homogenization approach applies in 3D.

We will consider microstructures generated from randomly located non overlapping cylinders with variable radii.

Different distributions of cylinders will lead to different EC losses since field distribution highly depends on the microstructure. A square structure of length $L_{1}=200 \mu \mathrm{m}$ is considered. The following constraints have been used to generate the microstructures: the distance from a cylinder to the boundary satisfies $D_{1} \geq 1 \mu \mathrm{m}$ and the distance between two cylinders satisfies $D_{2} \geq 2 \mu \mathrm{m}$. One realization of a random microstructure is shown in Fig. 11.

For such microstructures, the EC loss density estimates as defined in Eqs. (7) and (8) are difficult to use since the shape factor $\mathbf{K}$ needs to be defined for microstructures with cylinders with variable radii. A simple approach would be to choose the average radius $\bar{R}$ in Eq. (6), but it would not accurately consider the fact that cylinders with bigger radii have a bigger influence on EC losses than cylinders with smaller radii. A finer approach is detailed hereafter.

From Eqs. (6), (7), and (8), it appears that EC loss estimates are proportional to $\xi \cdot R^{2}$ for square array of cylinders (with same size) which can be rewritten as $\xi \cdot R^{2}=\pi R^{4} / L^{2}$ with $L$ the size of the periodic cell. Then, for microstructures made of $n$ cylinders with radii $R_{k}$ (with $k$ from 1 to $n$ ), and by making the assumption that each cylinder exhibits the same magnetic field distribution (same average field or same second order moment), then $\xi \cdot R^{2}$ can be extrapolated to

$$
\xi \cdot R^{2} \rightarrow \pi \frac{\sum_{k=1}^{n} R_{k}^{4}}{L_{1}^{2}} .
$$

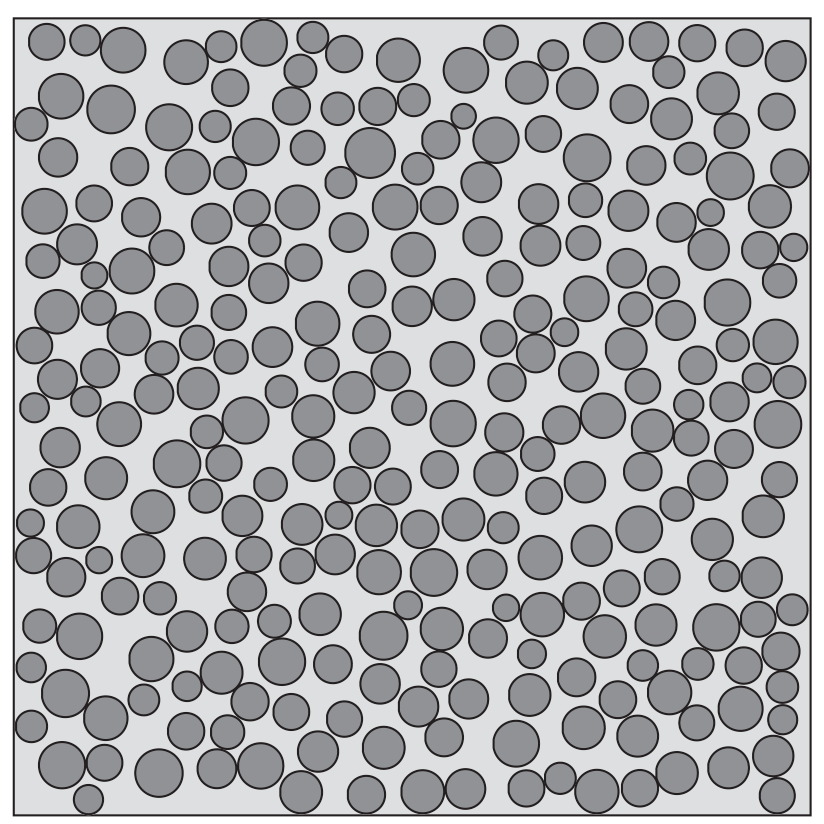

FIG. 11. One realization of SMC microstructure with a random distribution of non-overlapping cylinders with variable radii (filling factor $\xi=50 \%$ ).
With the definition of filling factor $\xi$ for such microstructures

$$
\xi=\pi \frac{\sum_{k=1}^{n} R_{k}^{2}}{L_{1}^{2}} .
$$

Then, the equivalent radius $\tilde{R}$ to use in Eqs. (7) and (8) is

$$
\tilde{R}^{2}=\frac{\sum_{k=1}^{n} R_{k}^{4}}{\sum_{k=1}^{n} R_{k}^{2}}
$$

If the microstructures are described with density probability functions for radii $p(r)$ (with $\int_{0}^{\infty} p(r) d r=1$ ), then the equivalent radius $R$ to use is

$$
\tilde{R}^{2}=\frac{\int_{0}^{\infty} p(r) r^{4} d r}{\int_{0}^{\infty} p(r) r^{2} d r}
$$

Multiple realizations of microstructures have been randomly generated with the following parameters:

- frequency $f=100 \mathrm{~Hz}, \mu_{i}=4000 \mu_{0}, \mu_{1}=\mu_{0}, \sigma_{i}=1.12$ $\times 10^{7} \mathrm{~S} / \mathrm{m}$, average flux density $B_{0}=1 \mathrm{~T}$

- filling factor $\xi$ : from $20 \%$ to $50 \%$ with steps of $5 \%$

- normal distribution of radii:

- fixed average radius $\bar{R}: 24 \mu \mathrm{m}$

- standard deviation $\sigma_{R}$ on the radius: from $0 \mu \mathrm{m}$ to $5 \mu \mathrm{m}$ with steps of $0.25 \mu \mathrm{m}$

For this kind of microstructure, the equivalent radius $R$ can be determined from Eq. (49) with an analytical formula

$$
\tilde{R}^{2}=\frac{\bar{R}^{4}+6 \sigma_{R}^{2}+\bar{R}^{2}+3 \sigma_{R}^{4}}{\bar{R}^{2}+\sigma_{R}^{2}} .
$$

250 realizations of random microstructures have been generated for each set of parameters. For each realization, the average magnetic field in the inclusions has been post-processed from the FEM results $\left(\left\langle H_{x}\right\rangle_{i, \text { FEM }},\left\langle H_{y}\right\rangle_{i, \text { FEM }}\right)$, as well as second order moment of the magnetic field $\left(\left\langle H_{x}^{2}\right\rangle_{i, \text { FEM }},\left\langle H_{y}^{2}\right\rangle_{i, \text { FEM }}\right)$, and the EC loss density values of the structure $\mathcal{U}_{\mathrm{FEM}}$.

Equations (7) and (8) have then been evaluated using the post-processed average and second order moment of magnetic field in order to build numerical estimates for EC loss density

$$
\left\{\begin{array}{l}
\mathcal{U}_{\mathrm{FEM}}^{-}=\frac{1}{2} \xi \pi^{2} \tilde{R}^{2} f \sigma_{i} \mu_{i}^{2}\left(\left\langle H_{x}\right\rangle_{i, \mathrm{FEM}}^{2}+\left\langle H_{y}\right\rangle_{i, \mathrm{FEM}}^{2}\right) \\
\mathcal{U}_{\mathrm{FEM}}^{+}=\frac{1}{2} \xi \pi^{2} \tilde{R}^{2} f \sigma_{i} \mu_{i}^{2}\left(\left\langle H_{x}^{2}\right\rangle_{i, \mathrm{FEM}}+\left\langle H_{y}^{2}\right\rangle_{i, \mathrm{FEM}}\right),
\end{array}\right.
$$

and using the equivalent radius $\tilde{R}$ defined by Eq. (50). 
Each realization has been checked independently and it has been found that the following inequality was verified every single time:

$$
\mathcal{U}_{\mathrm{FEM}}^{-} \leq \mathcal{U}_{\mathrm{FEM}} \leq \mathcal{U}_{\mathrm{FEM}}^{+} .
$$

This result seems to show that the EC loss density estimates built from average and second order moment of magnetic field are still bounds for random microstructures made of cylinders with random radii (it is demonstrated only in this paper for square array of cylinders).

Results presented hereafter are only for the microstructures with a filling factor of $50 \%$, which is a worst case scenario since the field distribution in inclusions is strongly heterogeneous compared to microstructures with lower filling factors.

Figure 12 shows the EC loss density as a function of radius standard deviation. It shows that the estimates, as defined in Eq. (51), seem to bound the EC loss density. Actually, every realization respected the bounding condition [Eq. (52)].

The decreasing and then increasing trend of EC loss density $\mathcal{U}_{\mathrm{FEM}}$, as exhibited on Fig. 12, can be explained as follows. First, FEM results show that the effective permeability $\tilde{\mu}$ decreases with standard deviation, which means that the amplitude of magnetic field in the inclusions decreases, which also means lower EC loss density. But at the same time, bigger standard deviation means that microstructures exhibit a few bigger inclusions that will generate more EC loss. The concurrence between these two processes explains the trend of EC losses.

Figure 13 shows the distribution of EC loss density estimates for the 250 microstructure realizations with standard deviation $\sigma_{R}=1.5 \mu \mathrm{m}$ (filling factor $\xi=50 \%$ ).

It shows that the distribution of EC loss density (and its estimates) roughly follows a normal distribution.

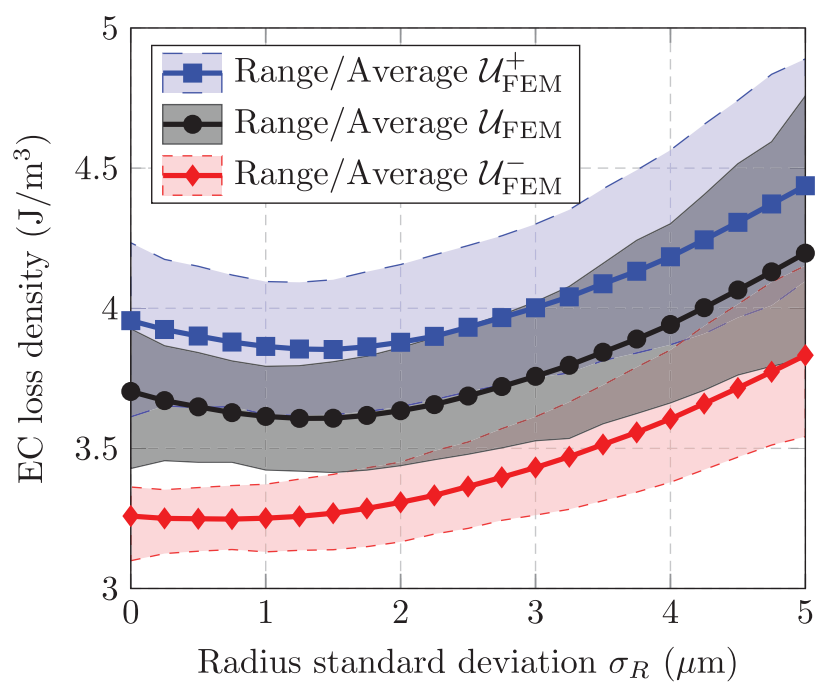

FIG. 12. EC loss density (FEM and estimates) as a function of radius standard deviation $\sigma_{R}$ (microstructures made of cylinders with radii chosen randomly from a normal distribution with an average radius $\bar{R}=24 \mu \mathrm{m}$ ). Filling factor $\xi=50 \%$. Dots represent the average values and the filled areas represent the range of obtained values over the 250 realizations for each standard deviation value.

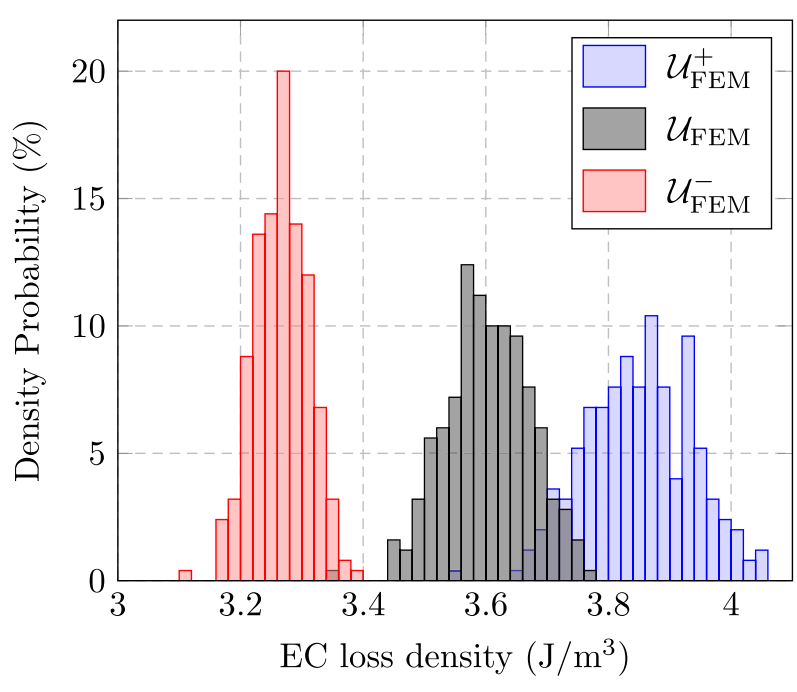

FIG. 13. EC loss density (FEM and estimates) distribution for $\sigma_{R}=1.5 \mu \mathrm{m}$ for 250 realizations of microstructure. Filling factor $\xi=50 \%$.

This numerical example tends to show that EC loss density estimate may be more general than only applicable to square array of cylinders or cubic arrays of spheres. In this example, these EC loss density estimates seem to bound the EC loss density for random distribution of non-overlapping cylinders with random radii.

\section{Application to experimental results}

In this section, the EC loss density estimates are compared with (semi-)analytical models and experimental approaches from the literature. de la Barrière $e t a l .{ }^{3}$ conducted experiments on two types of commercial SMCs. EC losses were separated from the total losses, and a (semi-)analytical model was developed to predict the EC losses. ${ }^{16,29}$ The microscopic images of the two SMCs are shown in Fig. 14.

The material parameters are detailed in Table II. The mean size represents the size of particles. The ferromagnetic inclusion material is pure iron, with density $\delta_{\mathrm{Fe}}=7870 \mathrm{~kg} /$ $\mathrm{m}^{3}$ and conductivity $\sigma_{\mathrm{Fe}}=9.93 \times 10^{6} \mathrm{~S} / \mathrm{m}$.

The above parameters are used as input for the EC loss estimate calculations. The volume fraction is obtained from the density ratio $\delta / \delta_{\mathrm{Fe}}, \xi_{\mathrm{A}} \approx 94.7 \%$, and $\xi_{\mathrm{B}} \approx 92.3 \%$. Since the volume fraction is high, the inclusions are considered as cubic and the effective permeability is determined using MG estimate. The corresponding EC loss density estimate is denoted $\mathcal{U}_{\mathrm{MG}}$. The results are plotted in Fig. 15.

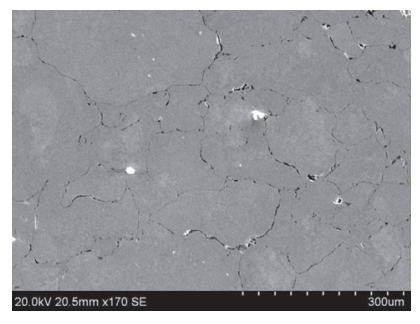

(a)

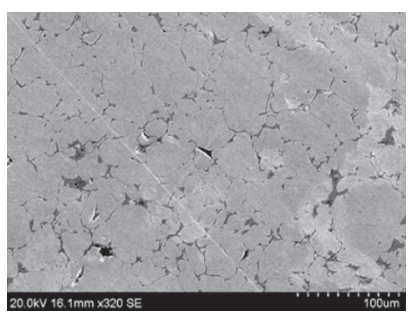

(b)
FIG. 14. Cross-sectional microscopic view of SMCs: (a) type A SMCs; ${ }^{29}$ (b) type B SMCs. ${ }^{3}$ 
TABLE II. Parameters for SMC A and B. ${ }^{3,29}$

\begin{tabular}{lccc}
\hline \hline & & SMC A & SMC B \\
\hline Relative permeability & $\mu_{r}$ & 450 & 110 \\
Electrical resistivity & $\rho(\Omega \mathrm{m})$ & $280 \times 10^{-6}$ & $7600 \times 10^{-6}$ \\
Density & $\delta\left(\mathrm{kg} / \mathrm{m}^{3}\right)$ & 7450 & 7260 \\
Mean size & $\langle s\rangle(\mu \mathrm{m})$ & 114 & 29.5 \\
\hline \hline
\end{tabular}

The proposed approach from MG permeability estimate provides a very satisfying result with respect to the experimental measurements. The approximation is comparable to that obtained with the "distribution" approach. ${ }^{29}$ The main difference between the two modelling approaches is that, while the "distribution" model requires a detailed analysis of the microstructure through micrographic analysis, data acquisition, and processing procedures, the model proposed in this paper is based on a statistical interpretation of the microstructure, leading to the application of a simple formula. Besides, the model is not restricted to a $2 \mathrm{D}$ view of the material, but

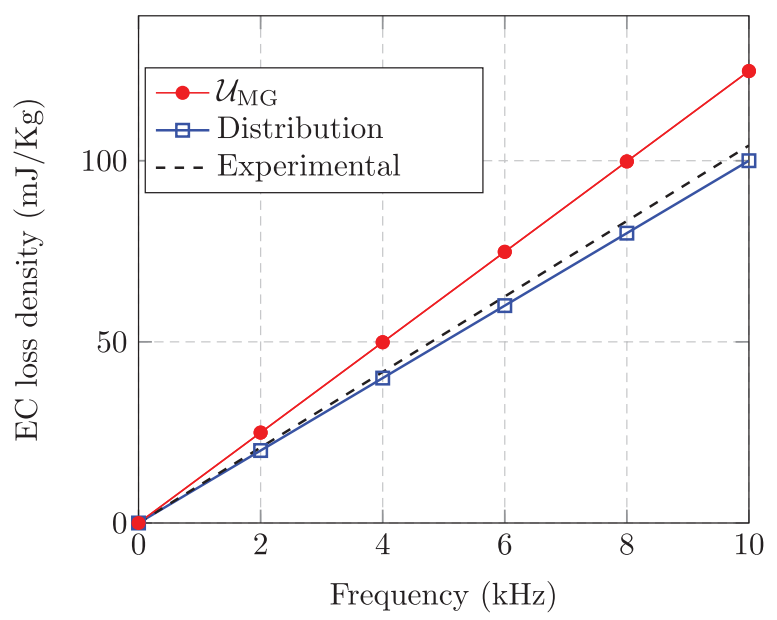

(a)

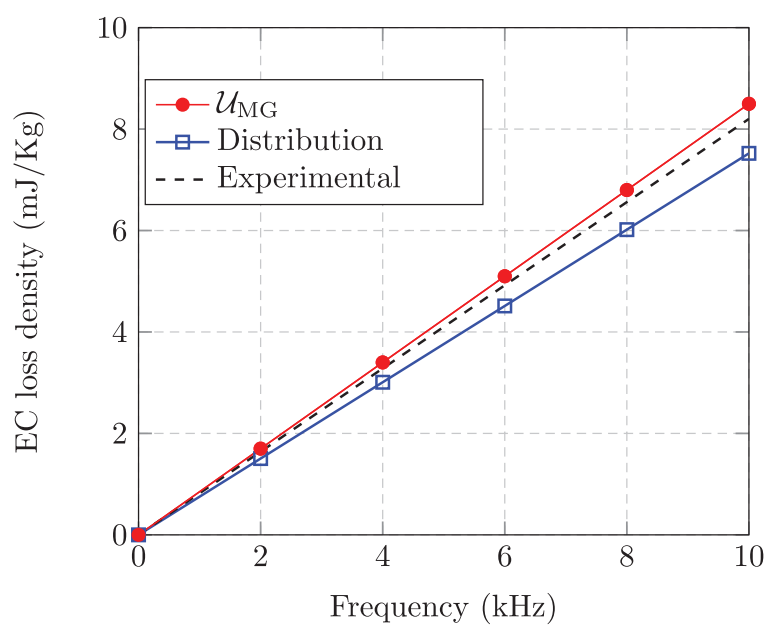

(b)

FIG. 15. Comparison of EC loss density from different models with experimental results. (Sinusoidal polarization, peak value: $1 \mathrm{~T}$ ). "Experimental" results are from Ref. 3; "distribution" results are the prediction from Ref. 29 considering the distributions of cross sections. encompasses 3D microstructures, making it a more generic tool for the description of magnetic composites.

\section{CONCLUSION}

It is demonstrated in this paper that average and second order moment of magnetic field can be used to build simple bounds on EC losses in composite materials with square lattice of cylindrical inclusions or cubic lattice of spherical inclusions, at low frequencies. Generic bounds are predicted from the average and second order moment of magnetic field approaches. The bounds may not be optimal, but can be used as relatively accurate estimates.

A generalization is proposed for a random distribution of (non-overlapping) cylinders, showing that average and second order moment of the magnetic field provide lower and upper estimates on EC losses.

The key point for evaluating these bounds and estimates is to know accurately the effective permeability which enables to retrieve the average and second order moment of the magnetic field.

A useful feature of this model is that EC distribution is not needed to estimate EC losses which makes this model very simple to implement. It only relies on a homogenization model for the effective permeability. The two estimates obtained in the case of periodic microstructures of spherical (3D) or circular (2D) inclusions are usually close to each other, providing accurate values for EC losses as long as the effective permeability is estimated with good accuracy.

The model presented in this paper has been first validated by comparison to FEM results. It was then compared to experimental measurements from the literature with very satisfying agreement.

\section{ACKNOWLEDGMENTS}

Xiaotao Ren is a fellowship beneficiary with the cooperation program between CSC (China Scholarship Council) and Université Paris-Sud.

\section{APPENDIX A: SPHERICAL SYMMETRY}

This appendix aims at proving that the EC loss density due to a magnetic field can be decomposed as the addition of EC loss densities generated separately from the normal decompositions of the magnetic field.

The microstructure is a cubic lattice of spherical inclusions, shown in Fig. 1(a). Because of spatial periodicity, the whole structure can be represented by an elementary cubic cell. It contains a sphere and its surrounding matrix.

Magnetic field $\overline{\mathbf{H}}_{\mathrm{I}}$ is imposed along the $x$-direction on the cell: $\overline{\mathbf{H}}_{\mathrm{I}}=\left[\overline{H_{1}}, 0,0\right]^{t}$ with the superscript $t$ the transpose operator. At a point $P(\mathbf{x})$ with $\mathbf{x}=(x, y, z), \mathbf{H}_{\mathrm{I}}, \mathbf{E}_{\mathrm{I}}$ denotes the magnetic field and the electric field at that point, respectively.

The matrix is dielectric, so the eddy current in the matrix is negligible. According to the definition, the eddy current loss density of the cell writes 


$$
\mathcal{U}_{\mathrm{I}}=\frac{\left\langle\sigma \mathbf{E}^{2}\right\rangle}{2 f}=\xi \sigma_{i} \frac{\left\langle\mathbf{E}_{\mathrm{I}}^{2}\right\rangle_{\text {sphere }}}{2 f},
$$

where $f$ is the working frequency. $\xi$ is the volume fraction of the sphere. $\sigma_{i}$ denotes the electric conductivity of the sphere. $\langle\cdot\rangle_{\text {sphere }}$ indicates the volume average operator over the sphere

$$
\langle\cdot\rangle_{\text {sphere }}=\frac{1}{V_{\text {sphere }}} \int_{\text {sphere }} \cdot \mathrm{d} V .
$$

Because of symmetry, the average magnetic field in the inclusion will be in the $x$-direction

$$
\left\langle\mathbf{H}_{\mathrm{I}}\right\rangle_{\text {sphere }}=\beta \overline{\mathbf{H}}_{\mathrm{I}},
$$

where $\beta$ is a coefficient depending on the volume fraction and properties of materials. $\beta$ is a scalar since the constituent materials are isotropic.

Magnetic field $\overline{\mathbf{H}}_{\mathrm{II}}$ is imposed along the $y$-direction: $\overline{\mathbf{H}}_{\mathrm{II}}=\left[0, \overline{H_{2}}, 0\right]^{t}$. Denote $\mathbf{H}_{\mathrm{II}}(\mathbf{x}), \mathbf{E}_{\mathrm{II}}(\mathbf{x})$ receptively the magnetic field and the electric field at the point $P$. Similarly, the average magnetic field in the sphere is

$$
\left\langle\mathbf{H}_{\mathrm{II}}\right\rangle_{\text {sphere }}=\beta \overline{\mathbf{H}}_{\mathrm{II}}
$$

and the EC loss density is

$$
\mathcal{U}_{\text {II }}=\xi \sigma_{i} \frac{\left\langle\mathbf{E}_{\text {II }}^{2}\right\rangle_{\text {sphere }}}{2 f} .
$$

It is clear that

$$
\overline{\mathbf{H}}_{\mathrm{I}} \perp \overline{\mathbf{H}}_{\mathrm{II}} \Rightarrow \mathbf{H}_{\mathrm{I}} \perp \mathbf{H}_{\mathrm{II}} \quad \text { and } \mathbf{E}_{\mathrm{I}} \perp \mathbf{E}_{\mathrm{II}}
$$

and

$$
\overline{\mathbf{H}}_{\mathrm{I}} \perp \overline{\mathbf{H}}_{\mathrm{II}} \Rightarrow\left\langle\mathbf{H}_{\mathrm{I}}\right\rangle_{\text {sphere }} \perp\left\langle\mathbf{H}_{\mathrm{II}}\right\rangle_{\text {sphere }} .
$$

The perpendicular relationship between $\mathbf{E}_{\mathrm{I}}$ and $\mathbf{E}_{\mathrm{II}}$ is indicated in Fig. 16.

Now the magnetic field $\overline{\mathbf{H}}_{\mathrm{I}}$ and $\overline{\mathbf{H}}_{\text {II }}$ are imposed simultaneously, noted $\overline{\mathbf{H}}_{\mathrm{III}} \cdot \overline{\mathbf{H}}_{\mathrm{III}}=\left[\overline{H_{1}}, \overline{H_{2}}, 0\right]^{t}$.

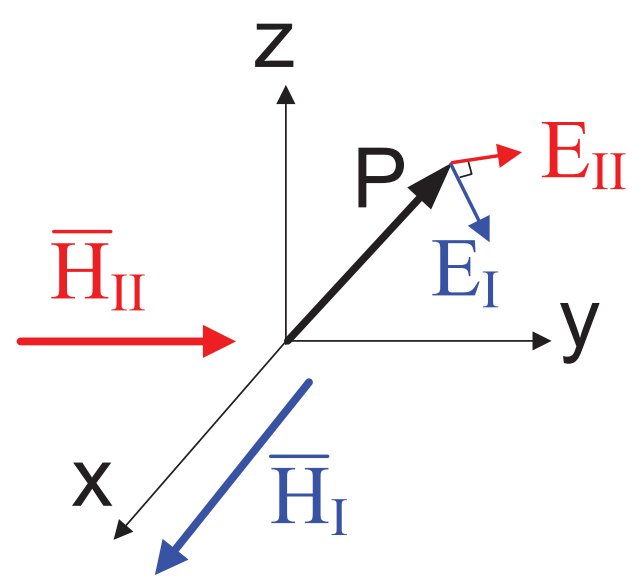

FIG. 16. Induced electric field
The electric field at point $P$ will be the addition of $\mathbf{E}_{\mathrm{I}}$ and $\mathbf{E}_{\mathrm{II}}$

$$
\mathbf{E}_{\text {III }}=\mathbf{E}_{\mathrm{I}}+\mathbf{E}_{\mathrm{II}} .
$$

The EC loss density of the cell writes

$$
\mathcal{U}_{\mathrm{III}}=\xi \sigma_{i} \frac{\left\langle\mathbf{E}_{\mathrm{III}}^{2}\right\rangle_{\text {sphere }}}{2 f} .
$$

Because $\mathbf{E}_{\mathrm{I}} \perp \mathbf{E}_{\mathrm{II}}$, then

$$
\mathbf{E}_{\mathrm{III}}^{2}=\mathbf{E}_{\mathrm{I}}^{2}+\mathbf{E}_{\mathrm{II}}^{2}
$$

so that

$$
\mathcal{U}_{\text {III }}=\mathcal{U}_{\mathrm{I}}+\mathcal{U}_{\mathrm{II}} .
$$

The magnetic field at point $P$ will be the addition of $\mathbf{H}_{\mathrm{I}}$ and $\mathbf{H}_{\mathrm{II}}$

$$
\mathbf{H}_{\text {III }}=\mathbf{H}_{\mathrm{I}}+\mathbf{H}_{\mathrm{II}} \text {. }
$$

The average magnetic field in the sphere is

$$
\begin{aligned}
\left\langle\mathbf{H}_{\text {III }}\right\rangle_{\text {sphere }} & =\left\langle\mathbf{H}_{\mathrm{I}}+\mathbf{H}_{\mathrm{II}}\right\rangle_{\text {sphere }} \\
& =\left\langle\mathbf{H}_{\mathrm{I}}\right\rangle_{\text {sphere }}+\left\langle\mathbf{H}_{\mathrm{II}}\right\rangle_{\text {sphere }} \\
& =\beta\left(\overline{\mathbf{H}}_{\mathrm{I}}+\overline{\mathbf{H}}_{\mathrm{II}}\right),
\end{aligned}
$$

which leads to the relationship

$$
\left(\left\langle\mathbf{H}_{\text {III }}\right\rangle_{\text {sphere }}\right)^{2}=\left(\left\langle\mathbf{H}_{\mathrm{I}}\right\rangle_{\text {sphere }}\right)^{2}+\left(\left\langle\mathbf{H}_{\text {II }}\right\rangle_{\text {sphere }}\right)^{2} .
$$

Because $\mathbf{H}_{\mathrm{I}} \perp \mathbf{H}_{\mathrm{II}}$, then

$$
\mathbf{H}_{\mathrm{III}}^{2}=\mathbf{H}_{\mathrm{I}}^{2}+\mathbf{H}_{\mathrm{II}}^{2}
$$

so that

$$
\left\langle\mathbf{H}_{\mathrm{III}}^{2}\right\rangle_{\text {sphere }}=\left\langle\mathbf{H}_{\mathrm{I}}^{2}\right\rangle_{\text {sphere }}+\left\langle\mathbf{H}_{\mathrm{II}}^{2}\right\rangle_{\text {sphere }} .
$$

The discussion is based on the two components of applied magnetic field. If the third component is not zero, the EC loss density generated by this component can be added directly to the final value. It is the same for the first (average field, vector) and second order moment of the magnetic field.

\section{APPENDIX B: DETERMINATION OF EFFECTIVE PERMEABILITY BY SERIES EXPANSIONS}

\section{Cylinder microstructure}

For cylinder microstructure, the effective permeability of the composite is generated by series expansion with the following formula: ${ }^{22}$

$$
\tilde{\mu}=\frac{1+\lambda(\xi) \xi}{1-\lambda(\xi) \xi} \mu_{1}
$$

where 


$$
\begin{aligned}
\lambda(\xi)= & \alpha+0.305827 \alpha^{3} \xi^{4}+\alpha^{3}\left(0.0935304 \alpha^{2}\right. \\
& +0.0133615) \xi^{8}+\alpha^{3}\left(0.0286042 \alpha^{4}\right. \\
& \left.+0.437236 \alpha^{2}+0.000184643\right) \xi^{12}+O\left(\xi^{16}\right),
\end{aligned}
$$

with

$$
\alpha=\frac{\mu_{i}-\mu_{1}}{\mu_{i}+\mu_{1}} .
$$

\section{Sphere microstructure}

For sphere microstructure, the effective permeability is generated by series expansion, which has the form ${ }^{25}$

$$
\tilde{\mu}=\left(1+\frac{3 \xi}{\gamma(\xi)}\right) \mu_{1},
$$

where

$$
\begin{aligned}
\gamma(\xi)= & -1 / R_{1}-\xi+1.3045 R_{3} \xi^{10 / 3}+0.0723 R_{5} \xi^{14 / 3} \\
& -0.5289 R_{3}^{2} \xi^{17 / 3}+0.1526 R_{7} \xi^{6}+O\left(\xi^{7}\right),
\end{aligned}
$$

with

$$
R_{n}=\frac{n\left(\mu_{1}-\mu_{i}\right)}{(n+1) \mu_{1}+n \mu_{i}} .
$$

\section{${ }^{1}$ G. Bertotti, IEEE Trans. Magn. 24, 621 (1988).}

${ }^{2}$ H. Shokrollahi and K. Janghorban, J. Mater. Process. Technol. 189, 1 (2007).

${ }^{3}$ O. de la Barrière, C. Appino, F. Fiorillo, C. Ragusa, H. B. Ahmed, M. Gabsi, F. Mazaleyrat, and M. LoBue, J. Appl. Phys. 109, 07A317 (2011).

${ }^{4}$ K. J. Sunday and M. L. Taheri, Met. Powder Rep. 72, 425-429 (2016).

${ }^{5}$ B. Yang, X. Li, R. Guo, and R. Yu, Mater. Des. 121, 272 (2017).
${ }^{6}$ J. Wang, X. Fan, Z. Wu, and G. Li, J. Mater. Sci. 52(12), 7091 (2017).

${ }^{7}$ T. Gheiratmand, H. M. Hosseini, and S. S. Reihani, J. Magn. Magn. Mater. 429, 241 (2017).

${ }^{8}$ G. Zhao, C. Wu, and M. Yan, J. Alloys Compd. 710, 138 (2017).

${ }^{9}$ Y. Cui and G. Kliman, in 39th IAS Annual Meeting Record of the 2004 IEEE Industry Applications Conference (IEEE, Seattle, WA, USA, 2004), Vol. 1, pp. 546-551.

${ }^{10}$ O. Bottauscio and A. Manzin, J. Comput. Phys. 253, 1 (2013).

${ }^{11}$ I. Niyonzima, R. V. Sabariego, P. Dular, and C. Geuzaine, IEEE Trans. Magn. 50, 61 (2014).

${ }^{12}$ L. Rayleigh, Philos. Mag. 34, 481 (1892).

${ }^{13}$ A. Sihvola, Electromagnetic Mixing Formulas and Applications (Institution of Electrical Engineers, 1999).

${ }^{14} \mathrm{G}$. W. Milton, The Theory of Composites (Cambridge University Press, 2002).

${ }^{15}$ X. T. Ren, R. Corcolle, and L. Daniel, Eur. Phys. J.: Appl. Phys. 73, 20902 (2016).

${ }^{16}$ O. de la Barriere, M. LoBue, and F. Mazaleyrat, IEEE Trans. Magn. 50, 1 (2014).

${ }^{17}$ X. Ren, R. Corcolle, and L. Daniel, IEEE Trans. Magn. 52(12), 1 (2016).

${ }^{18}$ R. Lipton, J. Appl. Phys. 96, 2821 (2004).

${ }^{19}$ R. Corcolle, L. Daniel, and F. Bouillault, J. Appl. Phys. 105, 123913 (2009)

${ }^{20}$ S. K. Mukerji, M. George, M. B. Ramamurthy, and K. Asaduzzaman, Prog. Electromagn. Res. B 7, 117 (2008).

${ }^{21} \mathrm{O}$. Wiener, Die theorie des mischkörpers für das feld der stationären strömung. 1. abhandlung: Die mittelwertsätze für kraft, polarisation und energie (B. G. Teubner, 1912).

${ }^{22}$ Y. A. Godin, J. Math. Phys. 54, 053505 (2013).

${ }^{23}$ J. D. Jackson, Classical Electrodynamics (John Wiley \& Sons Ltd., 1962), p. 67.

${ }^{24}$ K. T. Tang, Mathematical Methods for Engineers and Scientists 3 (Springer, 2007), pp.196-202.

${ }^{25}$ J. Lam, J. Appl. Phys. 60, 4230 (1986).

${ }^{26}$ R. C. McPhedran and D. R. McKenzie, Proc. R. Soc. London, Ser. A 359, 45 (1978).

${ }^{27}$ J. C. Maxwell-Garnett, Philos. Trans. R. Soc. London, Sect. A 203, 385 (1904).

${ }^{28}$ L. Daniel and R. Corcolle, IEEE Trans. Magn. 43, 3153 (2007).

${ }^{29}$ O. de la Barrière, C. Appino, F. Fiorillo, C. Ragusa, M. Lecrivain, L. Rocchino, H. B. Ahmed, M. Gabsi, F. Mazaleyrat, and M. LoBue, IEEE Trans. Magn. 49, 1318 (2013). 